\title{
O Apoio Multicritério à Decisão na avaliação de candidatos
}

\author{
Luís Alberto Duncan Rangel ${ }^{a, *}$, Luiz Flávio Autran Monteiro Gomes ${ }^{b}$ \\ a,**duncan@metal.eeimvr.uff.br, UFF, Brasil \\ bautran@ibmecrj.br, lbmec/RJ, Brasil
}

\begin{abstract}
Resumo
Os métodos de Apoio Multicritério à Decisão aplicam-se sempre que se deseje selecionar, ordenar, classificar ou descrever alternativas presentes em um processo decisório na presença de múltiplos critérios - quantitativos e qualitativos. Este artigo aborda o emprego dos métodos UTA e UTA-CR para a determinação das funções de utilidade dos critérios empregados para avaliar o ingresso de candidatos ao curso de graduação de Engenharia Metalúrgica da Universidade Federal Fluminense. Após apresentarem-se os métodos e o problema estudado, mostra-se, por uma análise dos resultados, que as funções de utilidade dos critérios obtidos com o método UTA-CR estão mais próximas das preferências dos decisores do que aquelas obtidas com o método UTA. Observa-se ainda que, pelo método UTA-CR, não há necessidade de realizar-se a análise de pós-otimização feita no método UTA, pois, com apenas uma única implementação de um programa linear, os resultados obtidos são melhores que os obtidos pelo método UTA. Palavras-chave
\end{abstract}

Apoio Multicritério à Decisão. Métodos de utilidade aditiva. Função de utilidade. Avaliação de candidatos.

\section{Introdução}

Os métodos de Apoio Multicritério à Decisão (AMD) são aplicados em inúmeras áreas em que se queira selecionar, ordenar, classificar ou descrever alternativas presentes em um processo decisório na presença de múltiplos critérios (ROY; BOUYSSOU, 1993; ROMERO, 1993; VINCKE, 1989). Muitas classificações são empregadas para designar os métodos do AMD. Dentre estas, a de maior repercussão é a que subdivide os métodos da Escola Americana e os métodos da Escola Francesa, também designada Escola Europeia. Existem outros métodos multicritério que, no entanto, não se enquadram exclusivamente dentro de uma dessas duas escolas (GOMES, 2006; BELTON; STEWART, 2002; BARBA-ROMERO; POMEROL, 1997). Como exemplos de métodos multicritério que contêm elementos técnicos dessas duas escolas, podem-se citar o Método TODIM (GOMES; RANGEL, 2007; GOMES; ARAYA; CARIGÑANO, 2004) e o Método MACBETH (BANA e COSTA; CORTE; VANSNICK, 2005).

Os métodos da Escola Francesa utilizam a noção de relação de superação, possuindo duas vertentes principais: os métodos ELECTRE e os métodos PROMÉTHÉE (VINCKE, 1989; ROY, 1991; ROY; BOUYSSOU, 1993; SCHÄRLIG, 1996; BRANS; VINCKE; MARESCHAL, 1986; BARBA-ROMERO; POMEROL, 1997).

Os métodos multicritério da Escola Americana, por sua vez, têm como fundamentação teórica a noção de agregarem-se todas as informações acerca do problema que se pretende resolver por meio de uma grande síntese. Exemplos significativos desses métodos são a Teoria de Utilidade Multiatributo (KEENEY; RAIFFA, 1993) e os métodos de análise hierárquica; dentre estes, o mais popular é, indiscutivelmente, o método AHP (SAATY, 1994). Em particular, a Teoria da Utilidade Multiatributo (conhecida também por MAUT Multiattribute Utility Theory) parte de suposições de racionalidade - como o respeito à transitividade estrita - e decorre das bases axiomáticas da teoria da utilidade esperada, propostas por Von-Neumann e Morgenstern (1953). Além disso, a MAUT não admite alguma possível incomparabilidade entre alternativas em um processo decisório (KEENEY; RAIFFA, 1993). Por conseguinte, pode-se afirmar que os métodos multicritério calcados na MAUT 
possuem uma base axiomática mais sólida do que os métodos que lançam mão da relação de superação. 0 método multicritério UTA (JACQUET-LAGRĖZE; SISKOS, 1982; ZOPOUNIDIS; DIMITRIS, 1998) é um exemplo de aplicação da MAUT.

Para a construção da função de utilidade multiatributo a ser aplicada a um determinado problema decisório, torna-se necessária a representação das funções de utilidade parciais. Estas são, na verdade, funções matemáticas que descrevem as performances de cada alternativa com relação às preferências dos agentes de decisão, para cada um dos critérios ou atributos daquele problema.

Este artigo faz uso de métodos do AMD para a determinação das funções de utilidade dos critérios empregados para avaliar o ingresso de candidatos no curso de graduação de Engenharia Metalúrgica da Universidade Federal Fluminense (UFF), localizado na cidade de Volta Redonda, RJ. Dois métodos foram utilizados na obtenção daquelas funções: o método UTA, em sua formulação clássica (JACQUETLAGRĖZE; SISKOS, 1982), e uma variante deste, o Método UTA-CR (RANGEL, 2002). Ambos os métodos têm por objetivo a Problemática P $\gamma$ (ROY; BOUYSSOU, 1993; GOMES; ARAYA; CARIGÑANO, 2004), isto é, de ordenação das alternativas em um problema de AMD. As ordenações dos candidatos são buscadas, também, pelas universidades, ao realizarem os respectivos vestibulares visando o ingresso dos melhores alunos. Desta forma, no estudo de caso apresentado, baseado nas pesquisas realizadas, além de se determinarem as funções de utilidades dos critérios, por meio do emprego dos métodos UTA e UTA-CR, obtiveram-se, também, as ordenações aproximadas dos candidatos. Assim, espera-se, com este estudo, contribuir efetivamente para a melhoria do processo do vestibular, uma vez que estas avaliações podem ser realizadas em anos seguintes, verificando os desempenhos dos candidatos, para aquelas mesmas provas, por meio das funções de utilidades, além das comparações das funções de utilidade dos critérios.

Em seguida, faz-se uma breve descrição da UFF e como se efetua o ingresso de alunos em seus diversos cursos de graduação.

\section{0 Contexto do estudo}

A UFF foi criada em 18 de dezembro de 1960, na cidade de Niterói, RJ. Originou-se a partir da união de cinco faculdades federais, três estabelecimentos de ensino estaduais e duas faculdades particulares sediadas naquele município.
Atualmente, a estrutura acadêmica da UFF fundamenta-se em quatro centros universitários: Centro de Ciências Médicas; Centro de Estudos Gerais; Centro de Estudos Sociais Aplicados, e Centro Tecnológico. A UFF tem aproximadamente 28.386 alunos matriculados e oferece 58 cursos de graduação, 43 programas de pós-graduação stricto sensu (21 em nível de mestrado e doutorado, $22 \mathrm{em}$ nível somente de mestrado), 96 programas de pósgraduação lato sensu e 115 cursos de extensão. 0 corpo docente compreende 2.466 professores: 1.015 doutores, 729 mestres, 262 especialistas e 460 graduados (UFF, 2006a).

A UFF oferece inúmeros cursos em diversos municípios do Estado do Rio de Janeiro, mas a concentração maior de cursos é na cidade de Niterói, onde fica também a Reitoria da Universidade. Além disso, a UFF possui também um campus avançado, localizado na cidade de Oriximiná, no Estado do Pará.

0 ingresso de alunos aos diversos cursos de graduação da UFF é feito por meio de uma avaliação realizada em duas etapas. Na primeira etapa, os candidatos realizam uma prova de múltipla escolha de conhecimentos gerais, englobando as disciplinas de Língua Portuguesa e Literatura Brasileira, Língua Estrangeira, Matemática, Física, História, Geografia, Química e Biologia. Em uma segunda etapa, os alunos realizam provas discursivas de conhecimentos específicos, de acordo com o curso em que pretendem ingressar. Para o curso de Engenharia Metalúrgica, pertencente ao Pólo Universitário de Volta Redonda da UFF, na segunda etapa, os alunos realizam provas específicas de Matemática e de Física, além da elaboração de uma Redação (UFF, 2006b).

A próxima seção mostra o desenvolvimento teórico dos dois métodos empregados neste estudo. A terceira seção apresenta o estudo de caso realizado. $\mathrm{Na}$ quarta seção, apontam-se as conclusões.

\section{Desenvolvimento}

0 AMD engloba diferentes métodos que empregam funções de preferência, como, por exemplo, o método PROMETHEE 11 (BRANS; VINCKE; MARESCHAL, 1986), da Escola Francesa, e funções de utilidade, como, por exemplo, o método MAUT (KEENEY; RAIFFA, 1993), da Escola Americana. Nesta pesquisa, optou-se pelo uso dos métodos UTA e UTA-CR, que medem a utilidade da cada alternativa de forma indireta. Com isto, tornou-se desnecessário: (i) efetuar uma comparação "par a par" de cada candidato, o que seria indispensável caso se empregasse, alternativamente, um método 
da Escola Francesa do AMD ou mesmo um método de análise hierárquica, como o AHP (SAATY, 1994) na sua versão tradicional, e (ii) construir uma função de utilidade de forma direta, como se precisaria fazer no caso de se empregar diretamente a Teoria de Utilidade Multiatributo. Assim, a opção do emprego dos dois métodos UTA e UTA-CR permite que se determinem, de forma indireta, as funções de utilidades dos critérios (SISKOS; GRIGOROUDIS; MATSATSINIS, 2005).

Naturalmente, outros métodos do AMD - como o das Trocas Justas (Even Swaps) (HAMMOND; KEENEY; RAIFFA, 1998) ou o MACBETH (BANA e COSTA; CORTE; VANSNICK, 2005) - poderiam ter sido, alternativamente, empregados. No entanto, como a intenção dos autores foi comparar os métodos UTA e UTA-CR quando aplicados a uma mesma base de dados, não caberia neste artigo o emprego de outros métodos.

Os métodos empregados nesta pesquisa, o Método UTA (JACQUET-LAGRĖZE; SISKOS, 1982) e o Método UTA-CR (RANGEL, 2002), utilizam a programação linear para a obtenção das funções de utilidade dos critérios, de acordo com as preferências dos agentes de decisão. Pelo método UTA, obtêm-se as funções de utilidade a partir de uma ordenação do conjunto ou do subconjunto das alternativas, proposta a priori pelos agentes de decisão. Emprega-se esta informação como restrição em um problema de programação linear (PPL), que tem como função objetivo minimizar o somatório dos erros associados para a obtenção da ordenação das alternativas proposta a priori.

Pelo método UTA-CR, obtêm-se as funções de utilidade de modo similar ao método UTA. No entanto, em vez de fazer uso das preferências dos agentes de decisão em relação ao conjunto de alternativas, empregam-se as preferências desses agentes com relação ao conjunto de critérios considerados. Chega-se à ordenação das alternativas a ser empregada como restrição no Método UTA-CR, por meio de um método auxiliar de ordenação multicritério. Nesta pesquisa, não houve necessidade de empregar um método para obter a ordenação das alternativas, pois se empregou a classificação obtida pelos candidatos para ingresso no Curso de Engenharia Metalúrgica da UFF.

0 método UTA-CR propõe ainda a inclusão de novas restrições ao PPL, além daquelas empregadas no método UTA, com o objetivo de reduzir a região viável de busca da solução ótima. Utiliza-se no UTA-CR uma nova função objetivo, composta por três termos. 0 primeiro termo, idêntico ao do método UTA, visa obter a minimização do somatório dos erros associados à utilidade de cada alternativa, com a finalidade de se chegar à ordenação a priori fornecida pelos agentes de decisão. Diversamente, o segundo e o terceiro termos da função objetivo do método UTA-CR visam obter um afastamento mínimo dos pesos dos critérios, para mais e para menos, respectivamente (RANGEL, 2002).

\subsection{Método UTA}

A primeira publicação na literatura internacional acerca do método UTA (Utilité Additive) data de 1982 (JACQUET-LAGRÈZE; SISKOS, 1982). Este método da Utilidade Aditiva permite obter as funções de utilidade dos critérios presentes na análise, partindo dos seguintes elementos: a matriz de avaliação, que contém as performances das alternativas em relação ao conjunto de critérios - performances estas que são estimadas pelos agentes de decisão; os julgamentos destes agentes quanto às possíveis preferências e indiferenças das alternativas ou um subconjunto destas.

Pelo método UTA, obtêm-se as funções de utilidade dos critérios por meio da execução de diversos problemas de programação linear (PPL). 0 primeiro PPL tem como função objetivo minimizar os erros associados às utilidades das alternativas, de modo a se obter a mesma ordenação proposta a priori pelos agentes de decisão. Posteriormente, rodam-se outros PPL, tendo como objetivo determinar o valor máximo e o valor mínimo que os extremos das funções de utilidade podem assumir, respeitando-se um conjunto de restrições.

Como referido na seção 1 deste artigo, o método UTA calca-se nas bases axiomáticas da MAUT, para determinar as funções de utilidade sob a forma aditiva (KEENEY; RAIFFA, 1993; BELTON; STEWART, 2002).

A proposta original dos criadores do método UTA foi assim definida:

Tendo uma estrutura de preferência de pré-ordem $R(\succ, \sim)$, com “ $\succ$ ” significando preferência estrita $e$ “ ” a indiferença em um conjunto de alternativas ou ações, o ajuste da função de utilidade aditiva baseado em critérios múltiplos é obtida de tal modo que a estrutura de preferência resultante seja tão consistente quanto possível com a estrutura inicial (JACQUETLAGRĖZE; SISKOS, 1982, p. 152).

Para explicar o método UTA, considere-se primeiramente a análise do conjunto de alternativas $A$, em presença de somente um critério de decisão. Sob esta consideração, verifica-se ou a preferência $\left[a \succ b \Leftrightarrow g_{i}(a)>g_{i}(b)\right]$ ou a indiferença $\left[a \sim b \Leftrightarrow g_{i}(a)=g_{i}(b)\right]$ entre duas alternativas $a$ e $b$ pertencentes ao conjunto $A$, sendo que o símbolo “ $\succ$ ” expressa a preferência e o símbolo “ ” expressa 
a indiferença entre duas alternativas. Estas relações entre as alternativas definem uma relação de ordem no conjunto $A$.

Considere-se agora uma família de critérios gi, em que $i$ varia de 1 a $n$, que avalia o conjunto de alternativas $A$. Considere-se ainda a agregação de todos os critérios em um único critério de síntese, $U(g)=U\left(g_{1}, g_{2}, g_{3}, \ldots, g_{n}\right)$. Sob esta condição, analisando-se o conjunto de alternativas, obtém-se a relação de preferência estrita $P$ e a relação de indiferença I (ROY; BOUYSSOU, 1993; VINCKE, 1989). A relação $R$, em que $R=P \cup I$, define uma pré-ordem para o conjunto de alternativas, pois permite a preferência e a indiferença.

A função de utilidade (Equação 1) é aditiva, apresentando, portanto, a seguinte forma matemática:

$$
U[g(a)]=\sum_{i=1}^{n} u_{i}\left[g_{i}(a)\right]
$$

em que cada ui(gi) é a utilidade marginal do desempenho gi no critério $i$. Uma hipótese fundamental, que é preciso respeitar quando se aplica uma função de utilidade aditiva, é a condição de independência mútua dos critérios em função das preferências (CLEMEN; REILLY, 2001; KEENEY; RAIFFA, 1993).

Os valores extremos das funções de utilidade de cada critério isão representados por: gi ${ }^{*}$, para o valor mais alto, e $g i^{*}$, para o valor mais baixo. Considere-se também que as funções de utilidade marginais $u i$ de cada critério $i$ são funções monótonas crescentes ou decrescentes. Desta forma, normalizam-se as funções de utilidade dentro do intervalo $[0,1]$, obtendo-se (Equações 2 e 3):

$$
\sum_{i=1}^{n} u_{i}\left(g_{i}^{*}\right)=1
$$

$u_{i}\left(g_{i^{*}}\right)=0$, para todo $i$

A Equação 2 normaliza os valores máximos de todos os critérios presentes na análise, indicando que o somatório destes valores tem de ser igual à unidade. Já a Equação 3 atribui o valor zero ao valor inicial de cada função de utilidade.

Os agentes de decisão expressam as suas preferências e indiferenças em relação ao conjunto de alternativas $A$ ou a um subconjunto representativo de alternativas $A$, de modo a obter uma ordenação dessas alternativas. 0 modelo matemático do método UTA busca essencialmente chegar a esta mesma ordenação ou ao mais próximo possivel desta, de modo que a utilidade calculada para uma alternativa $U^{\prime}[g(a)]$ difira da verdadeira
$U[(g a)]$, de um erro $\sigma(a)$. Desta forma, obtém-se o cálculo para toda alternativa $a \in A^{\prime}$ da seguinte forma (Equação 4):

$U^{\prime}[g(a)]=U[g(a)]+\sigma(a)$

Para duas alternativas consecutivas, de acordo com o conjunto $A$, têm-se as relações de preferência (Equação 5) e indiferença (Equação 6):

$U^{\prime}[g(a)]-U^{\prime}[g(b)] \geq \delta \Leftrightarrow$ se o agente

de decisão indica $a P b$

$U^{\prime}[g(a)]-U^{\prime}[g(b)]=0 \Leftrightarrow$ se o agente

de decisão indica $a I b$

$\mathrm{Na}$ Equação 5, $\delta$ é um número real suficientemente pequeno e maior do que zero, empregado para separar significativamente duas classes da pré-ordem completa $R$. Os autores do método UTA sugeriram que o valor de $\delta$ deve necessariamente pertencer ao intervalo [1/10Q, $1 / \mathrm{Q}]$, sendo $\mathrm{Q}$ o número de classes de indiferença (JACQUET-LAGRĖZE; SISKOS, 1982). Assumindo a existência de transitividade, pressuposto básico da MAUT, o agente de decisão só precisará fazer $(m-1)$ comparações entre alternativas, onde $m$ corresponde ao número de alternativas de $A$.

Substituindo (4) em (5) e (6), obtêm-se (Equações 7 e 8):

$$
\begin{aligned}
& \sum_{i=1}^{n}\left\{u_{i}\left[g_{i}(a)\right]-u_{i}\left[g_{i}(b)\right]\right\}+\sigma(a)-\sigma(b) \geq \delta \Leftrightarrow a P b \\
& \sum_{i=1}^{n}\left\{u_{i}\left[g_{i}(a)\right]-u_{i}\left[g_{i}(b)\right]\right\}+\sigma(a)-\sigma(b)=0 \Leftrightarrow a I b
\end{aligned}
$$

As funções ui são consideradas como sendo lineares por intervalos. Para definir tais intervalos, o agente de decisão escolhe $\alpha i$ pontos do intervalo [gi*, gi*], em que a função ui está definida, sendo $g i^{*}$ e $g i$, respectivamente, os limites inferiores e superiores de cada critério $i$. Por meio de cálculos que empregam interpolação, determinam-se os valores de $u_{i}\left[g_{i}(a)\right]$ em função dos intervalos definidos pelos decisores.

As funções de utilidade empregadas neste método são monótonas. Desta forma, a hipótese de monotonicidade é satisfeita através do seguinte conjunto de restrições (Equação 9):

$u_{i}\left(g_{i}^{j+1}\right)-u_{i}\left(g_{i}^{j}\right) \geq s_{i}, j=1,2,3, \ldots,\left(\alpha_{i-1}\right), i=1,2,3, \ldots, n$

em que $s i>O$ é o limite de indiferença definido para cada critério $i$.

Por conseguinte, o primeiro problema de programação linear deste método tem como função objetivo a minimização do somatório dos erros $\sigma(a)$, 
associados às utilidades das alternativas pertencentes a $A$ ', sujeitos às seguintes restrições: preferência entre as alternativas (Equação 7); indiferença entre as alternativas (Equação 8); monotonicidade das funções de utilidade (Equação 9), e normalização das funções de utilidade (Equações 2 e 3 ). Além destas restrições, todos os problemas de programação linear exigem a condição de não negatividade de suas variáveis, representada pelas Equações 10 e 11.

$u_{i}\left(g_{i}^{j}\right) \geq 0$, para todo $i$ e $j$

$\sigma(a) \geq 0$, para todo $a \in A^{\prime}$

Desta forma, tem-se o [PPL1] do método UTA (Equação 12):

[PPL1] Min $F=\sum_{i=1}^{m} \sigma(a)$

Sujeito a:

$\sum_{i=1}^{n}\left\{u_{i}\left[g_{i}(a)\right]-u_{i}\left[g_{i}(b)\right]\right\}+\sigma(a)-\sigma(b) \geq \delta \Leftrightarrow a P b$

$\sum_{i=1}^{n}\left\{u_{i}\left[g_{i}(a)\right]-u_{i}\left[g_{i}(b)\right]\right\}+\sigma(a)-\sigma(b)=0 \Leftrightarrow a I b$

$u_{i}\left(g_{i}^{j+1}\right)-u_{i}\left(g_{i}^{j}\right) \geq s_{i}, j=1,2,3, \ldots,\left(\alpha_{i-1}\right), i=1,2,3, \ldots, n$

$\sum_{i=1}^{n} u_{i}\left(g_{i}^{*}\right)=1$

$u_{i}\left(g_{i^{*}}\right)=0$,para todo $i$

$u_{i}\left(g_{i}^{j}\right) \geq 0$, para todo $i$ e $j$

$\sigma(a) \geq 0$, para todo $a \in A^{\prime}$

As Equações 7 e 8 são mutuamente exclusivas no [PPL1] (Equação 12), uma vez que o método respeita a transitividade entre as alternativas. A implementação deste método não termina com a busca da solução do [PPL1] (Equação 12), pois outras soluções são pesquisadas no entorno desta solução. Desta forma, considerando $F^{*}$ como sendo a solução ótima deste problema e $k\left(F^{*}\right)$ como sendo uma folga, buscam-se outras soluções de modo a satisfazer esta nova restrição (Equação 13):

$F \leq F^{*}+k\left(F^{*}\right)$

em que $k$ é um número real suficientemente pequeno e maior do que zero. Os vértices deste novo poliedro correspondem às funções de utilidade, em que um ou mais critérios atingem um peso extremo máximo ou mínimo. Implementam-se mais dois PPL para cada critério: um buscando determinar o valor mínimo que o valor extremo da função de utilidade do critério pode assumir e outro buscando determinar o valor máximo que o valor extremo da função de utilidade do critério pode assumir (Equações 14 e 15):

[PPL2] Min $u_{i}\left(g_{i}^{*}\right)$, para $i=1,2,3, \ldots, n$

[PPL3] Max $u_{i}\left(g_{i}^{*}\right)$, para $i=1,2,3, \ldots, n$

Esta etapa é chamada de análise pós-otimização, no método UTA. Estes dois novos problemas de programação linear [PPL2] (Equação 14) e [PPL3] (Equação 15) possuem as mesmas restrições do [PPL1] (Equação 12), além da restrição (Equação 13).

Depois destas implementações, pelo método UTA (JACQUET-LAGRĖZE; SISKOS, 1982), calculam-se as médias dos valores obtidos pelas variáveis que representam as funções de utilidade marginais ui(gij) verificadas na análise de pós-otimização [PPL2] (Equação 14) e [PPL3] (Equação 15). Após estes cálculos, pode-se avaliar a $U(g(a))$ de todas as alternativas $a \in A$, sejam ou não estas alternativas do conjunto de referência $A$ :

\subsection{Método UTA-CR}

0 método UTA-CR (Utilité Aditive - Critérios) proposto por Rangel (2002) é uma variante do método UTA. Esse método da Utilidade Aditiva baseada nos Critérios busca obter melhores soluções para as funções de utilidade e, para isto, considera as preferências dos decisores não em relação ao conjunto de alternativas, mas considerando 0 conjunto de critérios.

A ordenação das alternativas a ser empregada como dado de entrada no método UTA-CR será a ordenação obtida com o auxílio de outro método de ordenação do Apoio Multicritério à Decisão, tendo como referência as preferências dos decisores em relação ao conjunto de critérios.

0 modelo matemático do método UTA-CR leva em consideração as preferências dos decisores em relação ao conjunto de critérios, através do emprego de dois novos termos na função objetivo, além do termo que visa minimizar o somatório dos erros associados à ordenação das alternativas. No método UTA-CR, os dois novos termos da função objetivo visam minimizar os erros associados para mais e para menos das preferências dos decisores em relação ao conjunto de critérios. A Equação (16) apresenta a função objetivo do método:

$\operatorname{Min} F=\sum_{a \in A^{\prime}} \sigma(a)+\sum_{i=1}^{n} \xi\left(u_{i-S U P}^{*}\right)+\sum_{i=1}^{n} \xi\left(u_{i-I N F}^{*}\right)$

Duas novas restrições são empregadas neste método, além das empregadas no [PPL1] 
(Equação 12): a restrição (Equação 17), que busca restringir os valores extremos de cada critério para mais, e a restrição (Equação 18), que busca restringir os valores extremos de cada critério para menos.

$u_{i}\left(g_{i}^{*}\right)-\left(u_{i-S U P}^{*}\right) \geq w_{i}$

$u_{i}\left(g_{i}^{*}\right)+\left(u_{i-I N F}^{*}\right) \leq w_{i}$

Desta forma, tem-se definido um novo modelo matemático que representa o método UTA-CR (RANGEL, 2002), por meio do [PPL4] (Equação 19):

[PPL4] Min $F=\sum_{a \in A^{\prime}} \sigma(a)+\sum_{i=-1}^{n} \xi\left(u_{i-S U P}^{*}\right)+\sum_{i=-1}^{n} \xi\left(u_{i-I N F}^{*}\right)(19)$

Sujeito a:

$\sum_{i=1}^{n}\left\{u_{i}\left[g_{i}(a)\right]-u_{i}\left[g_{i}(b)\right]\right\}+\sigma(a)-\sigma(b) \geq \delta \Leftrightarrow a P b$

$\sum_{i=1}^{n}\left\{u_{i}\left[g_{i}(a)\right]-u_{i}\left[g_{i}(b)\right]\right\}+\sigma(a)-\sigma(b)=0 \Leftrightarrow a I b$

$u_{i}\left(g_{i}^{j+1}\right)-u_{i}\left(g_{i}^{j}\right) \geq s_{i}, j=1,2,3, \ldots,\left(\alpha_{i-1}\right), i=1,2,3, \ldots, n$

$\sum_{i=1}^{n} u_{i}\left(g_{i}^{*}\right)=1$

$u_{i}\left(g_{i^{*}}\right)=0$, para todo $i$

$u_{i}\left(g_{i}^{j}\right) \geq 0$, para todo $i$ e $j$

$\sigma(a) \geq 0$, para todo $a \in A^{\prime}$

$u_{i}\left(g_{i}^{*}\right)-\left(u_{i-S U P}^{*}\right) \geq w_{i}$

$u_{i}\left(g_{i}^{*}\right)+\left(u_{i-I N F}^{*}\right) \leq w_{i}$

No método UTA-CR (RANGEL, 2002), não há necessidade de realizar a análise pós-otimização que é feita no método UTA (JACQUET-LAGRÈZE; SISKOS, 1982), pois, com apenas uma única implementação do [PPL4] (Equação 19), os resultados obtidos são melhores que os obtidos pelo método UTA (RANGEL et al., 2003; ARAYA et al., 2002; RANGEL, 2006; RANGEL et al., 2006).

\section{Estudo de caso}

0 método UTA e sua variante UTA-CR foram empregados para avaliar um subconjunto de alunos selecionados, que prestaram exame vestibular para ingressar no curso de Engenharia Metalúrgica da Universidade Federal Fluminense (UFF), para início em 2006.

A COSEAC (Coordenadoria de Seleção), vinculada à PROAC - Pró-Reitoria de Assuntos Acadêmicos - é o órgão responsável pela organização, pelo planejamento e pela execução dos concursos de seleção para ingresso nos cursos de graduação da UFF, enquanto os colegiados dos cursos de graduação definem as disciplinas que comporão as respectivas provas específicas, que são discursivas.

Anualmente, a UFF realiza uma avaliação para o ingresso de alunos em seus diversos cursos. Estas avaliações são feitas em duas etapas. Na primeira etapa, faz-se uma prova de múltipla escolha abordando diversas disciplinas: Língua Portuguesa e Literatura Brasileira; Língua Estrangeira; Matemática; Física; História; Geografia; Química, e Biologia. Na outra etapa, os alunos realizam provas discursivas abordando duas disciplinas de conhecimentos específicos, de acordo com o curso pretendido, além da elaboração de uma Redação, que é realizada por todos os alunos candidatos a todos os cursos. Para o curso de Engenharia Metalúrgica, na segunda etapa, os alunos realizam provas específicas de Matemática e de Física (UFF, 2006b).

Assim, nesta pesquisa, os órgãos competentes da UFF são os decisores e os quatro critérios considerados nesta pesquisa são: o número de questões certas dos candidatos na primeira fase e as três notas das respectivas provas discursivas de Redação, Física e Matemática, da segunda fase.

Esta pesquisa foi realizada selecionando-se 14 entre os 119 candidatos, classificados e não classificados, que prestaram exame para o curso de Engenharia Metalúrgica da UFF. Não foram considerados nesta avaliação os candidatos eliminados por ausência ou por nota. A ordenação dos candidatos seguiu o resultado fornecido pela UFF, isto é, a colocação que o candidato conseguiu obter.

Com a finalidade de obter os pesos dos critérios empregados pela UFF, um sistema de equações foi resolvido, utilizando-se como dado de entrada os desempenhos dos candidatos. Depois de determinados, os pesos foram normalizados e seus valores são apresentados a seguir: o peso do critério $\mathrm{C}_{1}$ (Número de questões certas da Primeira Fase) foi aproximadamente 0,067 ; o peso dos critérios $\mathrm{C}_{2}$ (Nota da Prova de Redação), $\mathrm{C}_{3}$ (Nota da Prova de Matemática) e $\mathrm{C}_{4}$ (Nota da Prova de Física) foi 0,311, igualmente para cada um deles.

A Tabela 1 apresenta os desempenhos dos alunos em relação aos quatro critérios empregados nesta pesquisa, isto é, a matriz de avaliação. Os candidatos selecionados foram os seguintes: $A_{1}, A_{5}$, $A_{10}, A_{20}, A_{30}, A_{40}, A_{50}, A_{60}, A_{70}, A_{80}, A_{90}, A_{100}, A_{110} e$ $A_{115}$. Os índices associados a cada alternativa $A_{j}$, em que $j$ varia de 1 a 119 , representam a ordenação dos candidatos na avaliação realizada pela UFF. 
Tabela 1. Matriz de avaliação.

\begin{tabular}{|c|c|c|c|c|c|}
\hline \multirow{2}{*}{ Candidatos } & \multicolumn{4}{|c|}{ Critérios } & \multirow{2}{*}{$\begin{array}{l}\text { Ordenação } \\
\text { final }\end{array}$} \\
\hline & $C_{1}$ & $\mathrm{C}_{2}$ & $\mathrm{C}_{3}$ & $\mathrm{C}_{4}$ & \\
\hline$A_{1}$ & 55 & 8,5 & 6,6 & 6,2 & 1 \\
\hline$A_{5}$ & 44 & 8,0 & 4,9 & 6,8 & 5 \\
\hline$A_{10}$ & 40 & 7,0 & 7,1 & 5,7 & 10 \\
\hline $\mathrm{A}_{20}$ & 51 & 7,0 & 1,6 & 5,3 & 20 \\
\hline $\mathrm{A}_{30}$ & 47 & 5,75 & 3,0 & 5,0 & 30 \\
\hline $\mathrm{A}_{40}$ & 38 & 7,5 & 3,1 & 3,5 & 40 \\
\hline$A_{50}$ & 36 & 7,75 & 2,8 & 2,7 & 50 \\
\hline$A_{60}$ & 40 & 5,5 & 4,2 & 2,0 & 60 \\
\hline $\mathrm{A}_{70}$ & 28 & 6,0 & 4,1 & 3,1 & 70 \\
\hline$A_{80}$ & 23 & 7,25 & 3,0 & 3,1 & 80 \\
\hline$A_{90}$ & 21 & 8,0 & 0,5 & 3,7 & 90 \\
\hline$A_{100}$ & 24 & 8,5 & 0,4 & 1,4 & 100 \\
\hline$A_{110}$ & 25 & 5,0 & 0,3 & 2,7 & 110 \\
\hline$A_{115}$ & 17 & 7,0 & 0,3 & 0,5 & 115 \\
\hline
\end{tabular}

A ordenação das alternativas representa a primeira restrição no problema de programação linear da formulação dos dois métodos. Esta restrição indica que a alternativa $A_{1}$ é preferivel à alternativa $A_{5}$ e que a alternativa $A_{5}$ é preferivel à alternativa $A_{10}$, e assim sucessivamente. Por considerar a transitividade entre as alternativas, não há necessidade de analisar relações entre alternativas não sucessivas, tais como $A_{1} \operatorname{com~} A_{10}$, nem $A_{70}$ com $A_{100}$. As outras restrições do modelo são: monotonicidade dos critérios; somatório dos valores extremos máximos de cada critério igual a uma unidade, e valores mínimos de cada critério iguais a zero. A última restrição do problema de programação linear diz respeito à não negatividade das variáveis.

0 valor do parâmetro $\delta$ empregado na implementação dos dois métodos foi de 0,01 e seguiu a recomendação dos autores do método UTA (JACQUET-LAGRÉZE; SISKOS, 1982), que definem o valor deste parâmetro pertencente ao intervalo $[1 / 10 Q, 1 / Q]$.

Assim, neste estudo de caso, cada alternativa foi considerada como sendo uma classe. Como o conjunto de referência possui 14 elementos, é este, portanto, o número de classes consideradas. Esta consideração em relação ao número de classes resulta em um modelo que não possui relação de indiferença entre as alternativas presentes na análise. Note-se que o valor nulo do parâmetro "s", adotado na implementação dos dois métodos, significa que as funções a serem determinadas pelos modelos matemáticos dos dois métodos podem ser paralelas ou coincidir com o eixo das abscissas.
0 valor da função objetivo obtido com a implementação do [PPL1] (Equação 12) foi nulo, significando que o modelo matemático do método UTA conseguiu representar perfeitamente as preferências dos decisores. Então, foi realizada a análise pós-otimização com a implementação dos problemas de programação linear [PPL2] (Equação 14) e [PPL3] (Equação 15), com a finalidade de determinar os valores médios das variáveis. Oito implementações foram realizadas e, assim, determinaram-se as funções de utilidade dos critérios presentes na análise. Os resultados obtidos com as implementações deste método são apresentados em forma de gráfico, por meio de linha tracejada na Figura 1.

Com a finalidade de comparar os resultados dos dois métodos, em uma segunda etapa desta pesquisa, foi realizada a implementação do método UTA-CR [PPL4] (Equação 19) empregando os mesmos valores dos parâmetros da implementação do método UTA. Os resultados obtidos com a implementação do método UTA são apresentados na Figura 1, através de linha contínua. Ressalta-se que, na implementação deste método, não há necessidade de se realizarem as oito implementações - quatro do [PPL2] (Equação 14) e quatro do [PPL3] (Equação 15). Em uma única implementação [PPL4] (Equação 19), os valores das variáveis são obtidos.

\section{Comparação dos resultados}

As grandes diferenças das funções de utilidade dos critérios, obtidas na implementação dos dois métodos, buscam representar da melhor forma possivel as preferências dos decisores (Figura 1). Nas implementações dos dois métodos, verificam-se as diferenças dos valores máximos obtidos para cada critério. Assim, no critério $C_{1}$ (Número de questões certas da Primeira Fase), o valor proposto foi 0,067; o valor obtido pelo método UTA foi 0,221 e o obtido pelo método UTA-CR foi 0,067 . Seguem os valores obtidos para cada critério, respectivos aos métodos UTA e UTA-CR: critério $\mathrm{C}_{2}$ (Nota da Prova de Redação) - 0,084 e 0,311; critério $C_{3}$ (Nota da Prova de Matemática) - 0,250 e 0,311; critério $C_{4}$ (Nota da Prova de Física) - 0,444 e 0,311.

Verifica-se, por meio dos resultados apresentados pelo método UTA que os valores dos pesos dos critérios estão bem diferentes dos propostos $a$ priori pelos decisores. Estes valores propostos foram $0,067,0,311,0,311$, e 0,311 , e os valores obtidos com o emprego deste método foram 0,221, 0,084, 0,250 e 0,444 , para os critérios $C_{1}, C_{2}, C_{3}$ e $C_{4}$, respectivamente. Apesar de o modelo matemático 
Critério $\mathrm{C}_{1}$

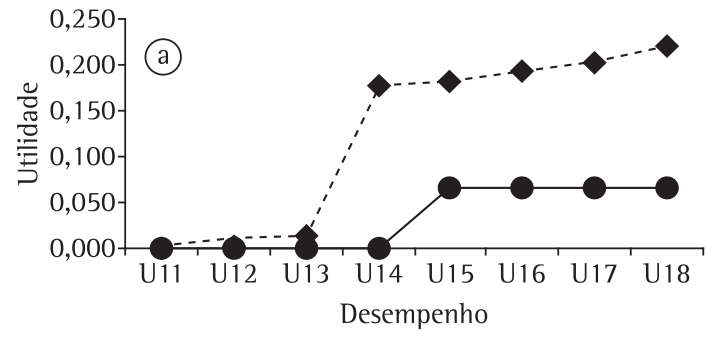

Critério $C_{3}$

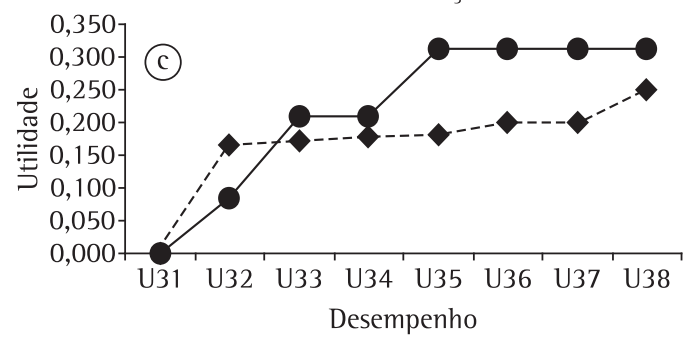

Critério $\mathrm{C}_{2}$

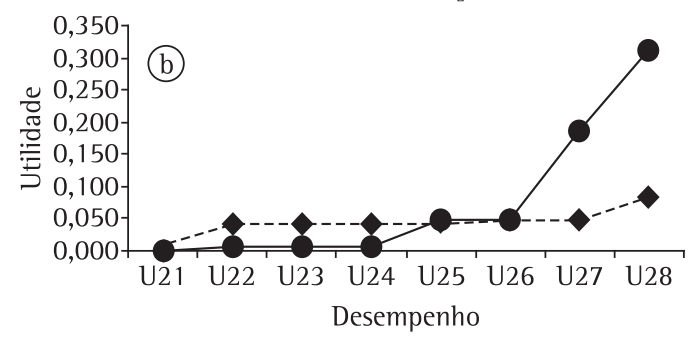

Critério $\mathrm{C}_{4}$

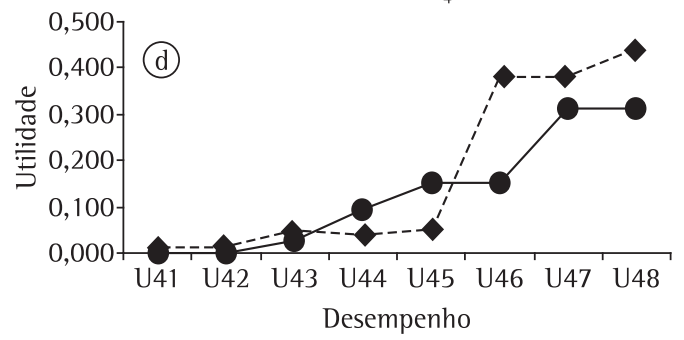

Figura 1. Funções de Utilidade dos Critérios $C_{1}, C_{2}, C_{3}$ e $C_{4}$. Método UTA (-) e Método UTA-CR (-๑).

conseguir representar as preferências dos decisores - pois o valor obtido pela função objetivo do [PPL1] (Equação 12) foi nulo -, isto não significa que estes valores representem perfeitamente as preferências dos decisores. Esse fato ocorre porque o problema de programação linear é degenerado.

Empregando-se o método UTA-CR (RANGEL, 2002) os valores obtidos dos pesos foram 0,067 , $0,311,0,311$ e 0,311 para os critérios $C_{1}, C_{2}, C_{3}$ e $\mathrm{C}_{4}$, respectivamente. Estes valores são os mesmos propostos a priori pelos decisores. 0 modelo matemático que representa este método busca obter os valores dos pesos com afastamento mínimo, tanto para mais como para menos, do valor proposto. Assim, este modelo determina as funções de utilidade dos critérios o mais próximo possivel das preferências dos decisores.

Com a finalidade de comparar os resultados das ordenações obtidas através dos dois métodos UTA e UTA-CR com a ordenação obtida do vestibular, foi realizado um estudo para determinar a correlação entre as ordenações. Em uma primeira etapa, determinou-se o coeficiente de correlação de Kendall (KENDALL; GIBBONS, 1990) entre a ordenação obtida através do vestibular da UFF e a obtida através do método UTA. 0 coeficiente de Kendall obtido foi igual a 0,8981 , indicando um alto grau de correlação entre as duas ordenações. Em uma segunda etapa, realizou-se o mesmo procedimento, para determinar a correlação entre a ordenação obtida pela a UFF e a obtida pelo método UTA-CR. Neste caso, o coeficiente de Kendall obtido foi igual a 0,8987, indicando também um alto grau de correlação. Desta forma, pode-se comprovar o alto grau de correlação obtida através dos métodos e a obtida através do vestibular. Através dos resultados, observa-se um aumento da correlação entre a ordenação obtida com o emprego do método UTA-CR e a ordenação obtida com o emprego do método UTA, quando comparadas.

Verifica-se que as funções de utilidades obtidas para os critérios são lineares por parte (Figura 1). Isto faz com que a ordenação dos candidatos obtida através destes métodos não seja tão precisa quanto a obtida através do vestibular. A ordenação obtida com o emprego dos métodos UTA e UTA-CR poderia ser melhorada com o aumento da qualidade das funções de utilidades, através do emprego de um maior número de pontos na definição destas funções; mas, por outro lado, um esforço maior teria de ser empregado, já que o número de variáveis de decisão nestes métodos é diretamente proporcional ao número de pontos e de critérios presentes na análise.

Sabe-se que todo processo de avaliação necessita de acompanhamento e estudo. No caso desta pesquisa, realizada com o emprego dos métodos UTA e UTA-CR para avaliar os candidatos ao curso de Engenharia Metalúrgica da UFF, a determinação das funções de utilidades baseada nos resultados do vestibular nos próximos anos irá propiciar um melhor acompanhamento do processo de avaliação do vestibular, uma vez que as funções de utilidades dos critérios poderão ser comparadas e analisadas. 


\section{Conclusão}

Dois métodos analíticos de Apoio Multicritério à Decisão foram empregados nesta pesquisa: o método UTA (JACQUET-LAGRĖZE; SISKOS, 1982) e uma variante deste, o método UTA-CR (RANGEL, 2002). 0 método UTA considera as preferências dos decisores em relação ao conjunto de alternativas para determinar as funções de utilidade, enquanto o método UTA-CR considera as preferências dos decisores em relação ao conjunto de critérios para, posteriormente, determinar uma ordenação das alternativas e, em seguida, determinar as funções de utilidade dos critérios.

As formas das funções de utilidade dos critérios obtidas com o emprego dos dois métodos são bem diferentes. Isto ocorre porque os problemas de programação matemática que representam os dois métodos são degenerados. 0 método UTA-CR emprega um número maior de restrições levando em consideração os pesos dos critérios e emprega, também, uma nova função objetivo.

Os pesos definidos pela universidade para os quatro critérios $C_{1}, C_{2}, C_{3}$ e $C_{4}$ neste processo de avaliação foram $0,067,0,311,0,311$ e 0,311 , respectivamente. Estes mesmos valores foram obtidos para os pesos critérios através do método UTA-CR. Já os pesos para os critérios determinados através do método UTA foram bem diferentes. Para os critérios $C_{1}, C_{2}, C_{3}$ e $C_{4}$ seus pesos foram 0,211 , 0,084, 0,250 e 0,444, respectivamente. Verifica-se, desta forma, que as funções de utilidade dos critérios obtidas com o método UTA-CR estão mais próximas das preferências dos decisores do que as obtidas com o método UTA, conforme os resultados das implementações e dos gráficos apresentados (Figura 1).

\section{Referências}

ARAYA, M. C. G. et al. Building the additive utility functions for CAD-UFRJ evaluation staff criteria. Annals of Operations Research, v. 116, n. 1-4, p. 271-288, 2002.

BANA e COSTA, C. A.; CORTE, J. M.; VANSNICK, J. C. On the mathematical foundations of MACBETH. In: FIGUEIRA, J.; GRECO, S.; EHRGOTT, M. (Ed.). Multiple criteria decision analysis. state of the art surveys. New York: Springer, 2005. p. 409-442.

BARBA-ROMERO, S.; POMEROL, J. C. Decisiones multicriterio: fundamentos teóricos y utilización práctica. España: Universidad de Alcalá, 1997. p. 420.

BELTON, V.; STEWART, T. J. Multiple criteria decision analysis: an integrated approach. Massachusetts: Kluwer Academic Publishers, 2002. p. 372.

BRANS, J. P.; VINCKE, P. H.; MARESCHAL, B. How to select and how to rank projects: the promethee methods.
European Journal of Operational Research, v. 24, n. 2, p. 228-238, 1986.

CLEMEN, R. T.; REILLY, T. Making hard decision with decisions tools. 2 ed. Rio de Janeiro: Thomson, 2001.

GOMES, L. F. A. M. Teoria da decisão. Rio de Janeiro: Thomson, 2006. p. 132.

GOMES, L. F. A. M.; ARAYA, M. C. G.; CARIGÑANO, C. Tomada de decisões em cenários complexos. Rio de Janeiro: Thomson, 2004. p. 168.

GOMES, L. F. A. M.; RANGEL, L. A. D. An application of the TODIM method to the multicriteria rental evaluation of residential properties. European Journal of Operational Research, v. 193, n. 2, p. 204-211, 2007. Disponivel em: $<$ http://www.sciencedirect.com>.

HAMMOND, J. S.; KEENEY, R. L.; RAIFFA, H. Even swaps: a rational method for making trade-offs. Harvard Business Review, v. 76, n. 2, p. 137-150, 1998.

JACQUET-LAGRĖZE, E.; SISKOS, J. Assessing a set of additive utility functions for multicriteria decision-making the UTA method. European Journal of Operational Research, n. 10, p. 151-164, 1982.

KEENEY, R. L.; RAIFFA, H. Decisions with multiple objectives: preferences and value tradeoffs. Cambridge: Cambridge University Press, 1993. p. 569.

KENDALL, M. G.; GIBBONS, J. D. Rank correlation methods. 5 ed. London: Edward Arnold, 1990. p. 260.

RANGEL, L. A. D. Determinação de funções de utilidade através das preferências dos decisores sobre o conjunto de critérios empregando o método UTA. Rio de Janeiro, 2002. Tese (Doutorado em Engenharia de Produção) Universidade Federal do Rio de Janeiro - UFRJ.

RANGEL, L. A. D. Determinação de funções de utilidade dos critérios empregados na avaliação de imóveis residenciais. In: CONGRESO IBERO-LATINOAMERICANO DE INVESTIGACIÓN OPERATIVA - CLAIO, 13, 2006. Anais... (CD-Rom).

RANGEL, L. A. D. et al. Avaliação da interiorização dos cursos da Universidade Federal Fluminense com o uso conjugado dos métodos UTA e MACBETH. Investigação Operacional, v. 23, n. 1, p. 49-70, 2003.

RANGEL, L. A. D. et al. A variant of the UTA method. In: INTERNATIONAL SYMPOSIUM ON MATHEMATICAL PROGRAMMING - ISMP, 19, 2006. Abstracts...

ROMERO, C. Teoría de la decisión multicriterio: conceptos, técnicas y aplicaciones. Madrid: Alianza, 1993. p. 195.

ROY, B. The outranking approach and the foundations of electre methods. Theory and Decision, v. 31, p. 49-73, 1991.

ROY, B.; BOUYSSOU, D. Aide multicritère à la décision: méthodes et cas. Paris: Econômica, 1993. p. 695.

SAATY, T. L. Fundamentals of decision making and priority theory: with the analytic hierarchy process. Pittsburgh: RWS Publications, 1994.

SCHÄRLIG, A. Pratiquer electre et prométhée: un complément à décider sur plusieurs critères. Lausanne: Press Polytechniques e Universitaires Romande, 1996. p. 173.

SISKOS, Y.; GRIGOROUDIS, E.; MATSATSINIS, N. F. UTA methods. In: FIGUEIRA, J.; GRECO, S.; EHRGOTT, M. (Ed.). Multiple criteria decision analysis: state of the art surveys. New York: Springer, 2005. p. 297-335.

UFF. Núcleo de Comunicação Social. Niterói: UFF, 2006. 
UFF. Coordenadoria de Seleção (COSEAC) da Pró-Reitoria de Assuntos Acadêmicos (PROAC) da UFF. Niterói: UFF, 2006. p. 64. (Edital de concurso e programa das disciplinas).

VINCKE, P. H. L'aide multicritère à la décision. Bruxelles: Éditions de l'Université de Bruxelles, 1989. p. 179.

VON-NEUMANN, J.; MORGENSTERN, 0. Theory of games and economic behavior. 3 ed. Princeton: Princeton University Press, 1953.

ZOPOUNIDIS, C.; DIMITRIS, A. 1. Multicriteria decision aid methods for the predictions of business failure. Dordrecht: Kluwer Academic Publishers, 1998. p. 171.

\section{Agradecimentos}

Os autores deste artigo agradecem ao $\mathrm{CNPq}$ Conselho Nacional de Desenvolvimento Científico e Tecnológico, através dos Projetos de Pesquisa 310603/2009-9, 502711/2009-4 e 306658/2004-6; à PROPP - Pró-Reitoria de Pesquisa e Pós-Graduação da Universidade Federal Fluminense, e ao Programa de Pós-Graduação e Pesquisa em Administração e Economia do lbmec/RJ.

\title{
Multicriteria Decision Aid in the evaluation of candidates
}

\begin{abstract}
The methods of multi-criteria decision aid are applied to problems of selection, ranking, classifying, and describing alternatives in the presence of multiple criteria, both quantitative and qualitative. This article tackles the problem of using the UTA and UTA-CR methods for determining the utility function of criteria used to evaluate candidates for the undergraduate program in metallurgical engineering in the Fluminense Federal University. After presenting the methods and outlining the problem, an analysis of the results shows that the utility functions of criteria obtained using UTA-CR are closer to decision-maker preferences than those obtained using UTA. It is also shown that, by using UTA-CR, it is not necessary to perform the post-optimization analysis that is required by the UTA method. In other words, implementation of a single linear program leads to results that are better than those obtained using UTA.
\end{abstract}

\section{Keywords}

Multicriteria Decision Aid. Additive utility methods. Utility function. Evaluation of candidates. 